\title{
Filtration behavior of silver
} nanoparticle agglomerates and effects of the agglomerate model in data analysis

\author{
Journal Article \\ Author(s): \\ Buha, Jelena; Fissan, Heinz; Wang, Jing \\ Publication date: \\ 2013-07 \\ Permanent link: \\ https://doi.org/10.3929/ethz-b-000070290
}

Rights / license:

In Copyright - Non-Commercial Use Permitted

Originally published in:

Journal of Nanoparticle Research 15(7), https://doi.org/10.1007/s11051-013-1709-z 


\title{
Filtration behavior of silver nanoparticle agglomerates and effects of the agglomerate model in data analysis
}

\author{
Jelena Buha $\cdot$ Heinz Fissan · Jing Wang
}

Received: 18 January 2013/Accepted: 6 May 2013/Published online: 20 June 2013

(C) Springer Science+Business Media Dordrecht 2013

\begin{abstract}
In many data evaluation procedures for particle measuring devices and in filtration models, spherical particles are assumed. However, significant fractions of aerosol particles are agglomerates of small primary spheres. The morphology of particles in filtration processes may not be known a priori and if the filtration data are processed with wrong assumption, errors can be induced. In this work, we have quantified such errors for the case of open-structured agglomerates. Filtration efficiency tests with polydisperse silver nanoparticle agglomerates and their sintered spheres were performed. After the sintering process, particles with a compact structure with the shape close to a sphere are obtained, which are referred to as sintered spheres in the present study. The testing method involved generation of particulate
\end{abstract}

Special Issue Editors: Mamadou Diallo, Neil Fromer, Myung S. Jhon

This article is part of the Topical Collection on Nanotechnology for Sustainable Development

J. Buha $(\bowtie) \cdot$ J. Wang $(\bowtie)$

Analytical Chemistry, Empa, 8600 Dübendorf,

Switzerland

e-mail: jelena.buha@empa.ch

J. Wang

e-mail: jing.wang@ifu.baug.ethz.ch

J. Buha $\cdot$ J. Wang

Institute of Environmental Engineering, ETH Zurich,

8093 Zurich, Switzerland forms, passing the particles through the testing section, and measurement of the particle number concentrations and size distributions before and after the filter. Measurements of the aerosols upstream and downstream of the filter were conducted using scanning mobility particle sizers (SMPS, TSI Inc.), which covered the rage from 10 to $480 \mathrm{~nm}$. Particles were additionally characterized from the electron microscopic images and the average primary particle size was determined to be $16.8 \mathrm{~nm}$. The number-size distribution curves were obtained and used for penetration calculation. The penetration was dependent on the particle size and morphology. Silversintered spheres were captured with a lower efficiency than agglomerates with the same mobility diameter because of the stronger interception effect for agglomerates. Data analysis of the number-size distribution for agglomerates was processed based on sphere assumption and using the model for openstructured agglomerates developed by Lall and Friedlander. The efficiencies based on total concentrations

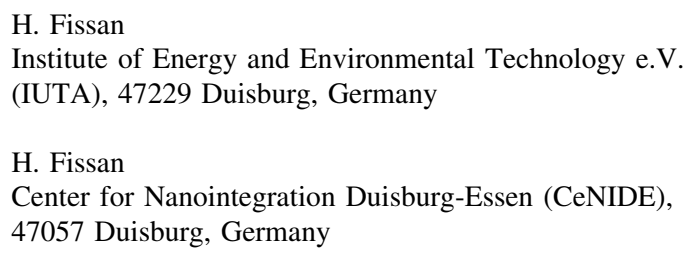


of number, surface and volume were affected when the agglomerate model was used. The effect was weakest for the total number efficiency and strongest for the total volume efficiency. Filtration efficiency curves for agglomerates were additionally plotted as a function of the volume equivalent diameter or number of primary particles per agglomerate, because the latter parameters provide more information for the agglomerate structure.

Keywords Nanoparticle agglomerates $\cdot$ Silver · Filtration · TEM $\cdot$ Mobility diameter

\section{Introduction}

Many engineered nanoparticles (NP) and natural aerosols exist in the form of NP agglomerates. Accordingly, filtration of NP agglomerates is gaining more attention (Fu et al. 1990; Lange et al. 1999; Kim et al. 2009a, b; Cena et al. 2012). An important group of agglomerates consist of clusters or chains of nanosize spherules with point contacts. They are often produced by high temperature processes leading to a solid particulate. It has been suggested that particle surface area is the most important parameter in terms of NP toxicity (Oberdörster et al. 2005). Silver agglomerates, among the others, are a health concern; they are expected to be more toxic than spherical silver particles with the same mass due to larger surface area. The additional reason for choosing silver agglomerates for this study is the extensive number of reports on their generation and properties and the advantage of easy control over the morphology by adjusting the sintering temperature ( $\mathrm{Ku}$ and Maynard 2006; Lall et al. 2006; Shin et al. 2009a, b).

There are some reports available quantifying the physical properties of agglomerates. Mobility is a fundamental property that can be readily measured and determines their transport and the kinetics of their growth (Sorensen 2011). While there have been a number of reports of the dynamic shape factor of regular 3-D structures such as ellipsoids, rods, and of multiplets (Kousaka et al. 1996; Song et al. 2005; Zelenyuk and Imre 2007), there have been few studies for larger agglomerates produced by high temperature processes. One of these was the study by Park et al. (2004) to measure the dynamic shape factor of diesel exhaust particles. They used a differential mobility analyzer (DMA) and electron microscopy (EM) to measure the dynamic shape factor, which is finally a function of only the mobility diameter and the volume equivalent sphere diameter. They were able to obtain dynamic shape factor measurements of diesel exhaust particulates with an uncertainty of $10-15 \%$ over a mobility size range from 50 to $220 \mathrm{~nm}$. In some studies, various predictions for the friction coefficient of large agglomerates were tested. In order to get reliable data, the friction coefficient of NP agglomerates as a function of the agglomerate size for agglomerates with primary sphere size in the free molecular regime was examined (Shin et al. 2009b).

So as to analyze agglomerates in detail, initial step is the determination of the primary particle size, which is an important structural parameter for open-structured agglomerates with most primary particles available for interaction with the suspending media and minimal necking among primary particles. It may be determined using electron microscopy, as presented in our study, or quasi-online using the instrument UNPA (Shin et al. 2010; Wang et al. 2010). The second important step is resolving the number of primary particles within the agglomerate. There have been a number of agglomerate studies on the relationship between the mobility radius and the radius of gyration and between the mobility radius and the number of primary particles in agglomerate. These include the studies for in-flame carbonaceous aerosols in the free molecular regime (Cai and Sorensen 1994), or an insightful review of other experimental studies covering a wide range of Knudson number (Wang and Sorensen 1999).

The present work focuses on filtration of agglomerates instead of detailed structural characterization. Particle collection in fibrous filters is a very complex problem in filtration theory, and therefore spherical particles are mainly considered. Nevertheless, realworld particles often have a complicated structure, which influences their deposition behavior. The study by Lange et al. (2000) showed that the concept of equivalent diameters can be used to predict filter penetration for nonspherical particles if relevant diameters have been measured, and if these are inserted into models for spherical particles. In a previous study by Lange et al. (1999), filter penetration was successfully predicted for the regime where diffusion and interception are the dominant deposition mechanisms. The measured penetrations agreed well with the calculations.

Air filtration tests are often performed by challenging the filter with testing aerosols and measuring the aerosol 
concentrations upstream and downstream of the filter using a CPC for total efficiency and a SMPS for number distribution as function of mobility diameter. Kim et al. (2009a) measured filtration efficiency for monodisperse silver NP agglomerates in standard fiberglass media. In contrast, we tested polydisperse silver NP agglomerates in nanofiber filters in the present study. Polydisperse aerosols are frequently used since one test can provide the filtration efficiency curve for a wide range of particle sizes (Song and Park 2006; Japuntich et al. 2007). This approach requires accurate measurement of the numbersize distribution of the aerosols. If polydisperse NP agglomerates are used as challenging aerosols in filtration experiments, the data evaluation for the measurement could be based on sphere assumptions or open-structured agglomerate assumptions, for example, using a model developed by Lall and Friedlander (2006). This model was validated and justified for openstructured silver agglomerates in the studies reported before (Shin et al. 2009a; Wang et al. 2010). We have examined the filtration data using silver NP agglomerates. The filtration efficiencies based on number, surface area and volume are further on computed with these different assumptions. Penetration of silver agglomerates through fibrous filters was predicted, using a model for spheres and suitable equivalent diameters for the agglomerates. The main results are presented in five folds: (1) Characterization of all silver particulate forms produced. (2) Experimental results using polydisperse aerosols, showing that the penetration of agglomerates was lower than that of sintered spheres with the same mobility diameter. (3) Modeling for filtration of sintered spheres and agglomerates and the comparison to the experimental results. (4) Analyses of the number-size distribution data based on sphere and open-structured agglomerate assumptions; the results show that using the correct structural parameters impacts calculation of the total filtration efficiency. (5) The filtration efficiency of agglomerates considered as function of structurerelated parameters, e.g., the volume equivalent diameter and number of primary particles per agglomerate.

\section{Materials, methods, and characterization}

\section{Experimental set-up}

Silver primary particles were generated by the evaporation and condensation method with silver slugs
(Alfa Aesar, $99.99 \%$ metal based) within an electric tube furnace. One silver slug, with the dimensions of $6.35 \mathrm{~mm}$ (diameter and length), was placed in a ceramic boat and positioned in the middle of the first tube furnace, where the silver was heated to $1150{ }^{\circ} \mathrm{C}$ and further condensation led to silver NP. The particle generation system was stable during our experiments. Particle concentration at the peak and the standard deviation of the size distribution measured with SMPS varied within $20 \%$. The peak location varied by a few nanometers.

Silver agglomerates were produced by passing primary particles through an agglomeration chamber to increase the residence time needed for coagulation to take place. Nitrogen gas was used as the carrier gas with a flow rate of $1.5 \mathrm{lpm}$. The general methodology that was employed is similar to the ones reported before (Ku and Maynard 2006; Lall et al. 2006; Shin et al. 2009a, b). Silver NP, collided and attached to each other in the agglomeration chamber leading to NP agglomerates made up of a few up to hundreds primary particles.

Downstream of the agglomeration chamber, the NP agglomerates were sintered in a second tube furnace. The agglomerates were coalesced at $600{ }^{\circ} \mathrm{C}$, which allowed direct comparison of the filtration behavior of the agglomerate to that of a sphere with the same mobility diameter and for the same material.

Schematic diagram of the experimental set-up is shown in Fig. 1. It consisted of an NP agglomerate generator (two electric tube furnaces), an agglomeration chamber (16 1 volume capacity), a nanometer aerosol sampler for TEM sampling, a Kr-85 neutralizer, two SMPS systems for size distribution measurements, and a filter penetration measurement system (multiple layers of nanofiber filters and the filter holder). The testing method involved generation of silver particles, passing the particles through the testing section, and measurement of the particle concentrations and size distributions before and after the filter. Size distributions of the primary particles, as well as silver agglomerates and sintered agglomerates upstream and downstream of the test filter, were measured by SMPS systems. Each of the particulate form (primary particles, silver agglomerates, sintered silver spheres) was further on collected for the offline electron microscopy analysis. Face velocity was controlled with the flow meter and kept constant $(5 \mathrm{~cm} / \mathrm{s})$ throughout all of the experiments. 
Fig. 1 Schematic of NP agglomerate synthesis and characterization set-up system

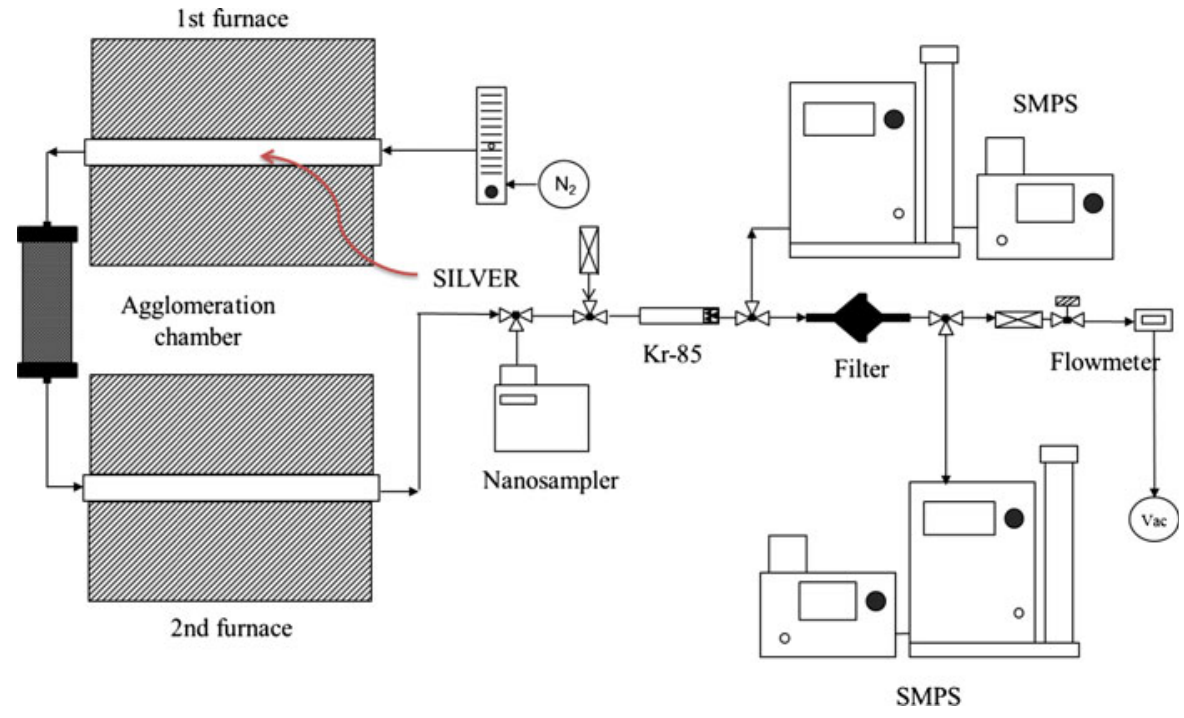

\section{Instrumentation}

The generated particles were characterized in the aerosol form by particle size distribution measurement and in the collected particle form by transmission electron microscopy (TEM). The particle size distribution was measured by an SMPS consisting of a DMA column (Model 3081, TSI, USA) and a condensation particle counter (CPC) (Model 3775, TSI, USA). The imaging was carried out using TEM (JEM-2200FS, JEOL, Japan). For TEM, the particles were collected on a copper grid covered by an amorphous carbon film with a nanometer aerosol sampler (NAS) (3089, TSI, USA).

\section{Results and discussion}

Characterization of silver agglomerates and sintered spheres

After producing silver NP from silver powder using the electric tube furnaces and the agglomeration chamber, SMPS measurements (Figs. 2, 4) and extensive electron microscopy studies were performed (Fig. 3). Size distributions of particles generated in the first furnace and measured at the outlet at room temperature (no agglomeration, no sintering) for three times, which qualitatively demonstrated the temporal stability and reproducibility. Respective SMPS results are presented in Fig. 2a, which give a representation of the primary particle size distribution since they do not adequately agglomerate before the agglomeration chamber. Primary particles were additionally identified from the TEM images of the open-structured silver agglomerates and their sizes were determined. The primary particle size was calculated out of 188 particles incorporated into agglomerates. It was ascertained that the average primary particles of silver agglomerates had an average diameter of $16.8 \mathrm{~nm}$, with a standard deviation of $3.75 \mathrm{~nm}$ as shown in Fig. 2b. Primary particles were polydispersed as can be seen on the graph. They were fitted well with a Gaussian distribution function, as shown in the inset. The studies reported before show that the primary particle size of silver agglomerates in diameter was $15 \mathrm{~nm}$ in Schmidt-Ott (1988) and $18.5 \mathrm{~nm}$ in Lall et al. (2006).

TEM analysis was performed to characterize the primary particles, the agglomerates and the sintered spheres formed after the sintering process in the second furnace. All samples were collected for a short period of time to avoid particle overlapping due to the high concentrations. Figure 3 a shows a TEM image of the primary spheres. Figure $3 b$ is showing the open-structured agglomerates, sampled after the agglomeration chamber and the second furnace at room temperature. The image of the compact-structured particles formed by the sintering process at $600{ }^{\circ} \mathrm{C}$ is presented in Fig. 3c. This is a representative image out of 20 taken, and we notice a general trend of small particles being attached to the surface. 

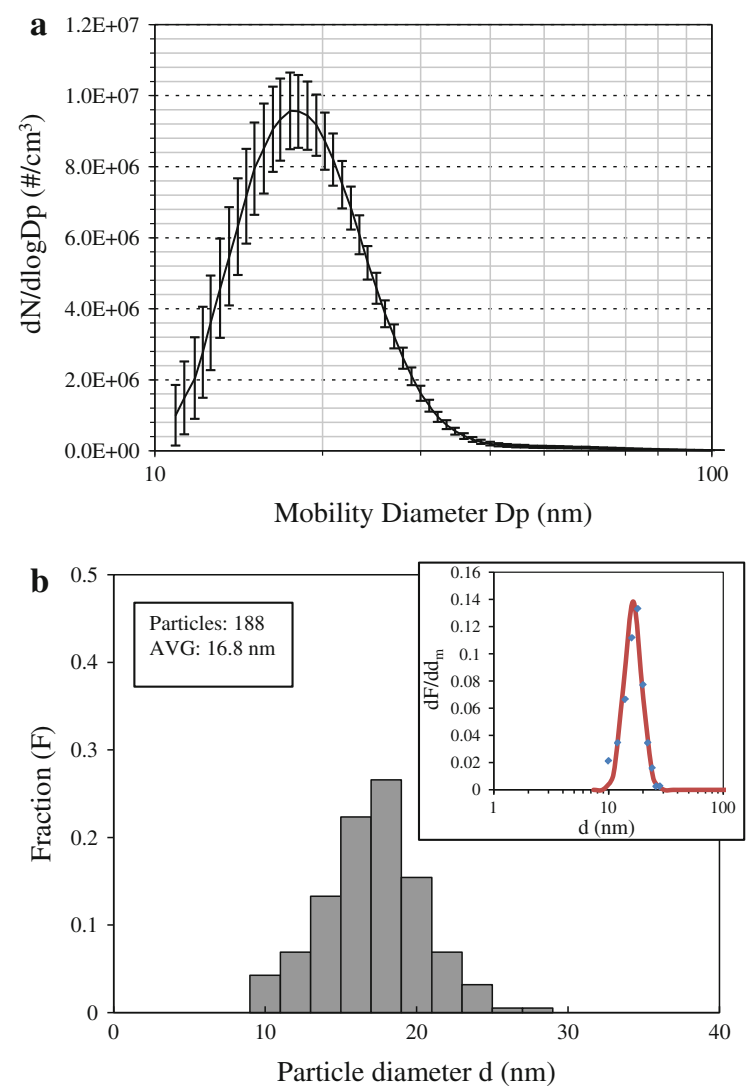

Fig. 2 a The size distributions of primary silver nanoparticles, produced after the first electrical tube furnace treatment at $1150{ }^{\circ} \mathrm{C}$ (SMPS results); b size distribution of the primary particles in open-structured agglomerates and fitted Gaussian distribution as inset

The melting temperature of silver is $962{ }^{\circ} \mathrm{C}$ which is higher than the temperature used in the sintering furnace. Thus, the sintered spheres are not totally coalesced, instead smaller particles are attached to the surface due to the heterogeneous coagulation. A number of previous studies (Schmidt-Ott 1988; Ku and Maynard 2006; Kim et al. 2009a; Cena et al. 2012) confirmed using SEM or low resolution TEM that the $\mathrm{Ag}$ particles sintered at $600{ }^{\circ} \mathrm{C}$ had shapes close to spheres. Our TEM analysis also showed the overall particle shape was close to a sphere, but the detailed structure of the small particles at the surface is for the first time reported. The detailed surface structure and the imperfect sphericity of the sintered particles have little effect on the filtration behavior, which is demonstrated by the good agreement between the theoretical model assuming perfect spheres and the experimental results in Sect. 3.3. Similarly, Kim et al. (2009a) and Cena et al. (2012) compared their filtration results using $\mathrm{Ag}$ particles sintered at $600{ }^{\circ} \mathrm{C}$ with models assuming perfect spheres. Significant size increase of spheres after the sintering process in the second tube furnace (Fig. 3c) is obvious when compared with the initial primary particles shown in Fig. 3a (scale bar is the same for all the images). Most likely, the grain boundary diffusion took place when spheres were formed out of the agglomerated NP during the sintering process. Primary size distribution was estimated from the TEM micrographs of silver agglomerates. During the collection of the silver NP agglomerates, directly after the agglomeration chamber, the sampling time was optimized to be able to distinguish the sizes and morphology of the agglomerates produced. Size of the particles determined from TEM images was in good agreement with the SMPS results obtained for primary particles (Fig. 2a).
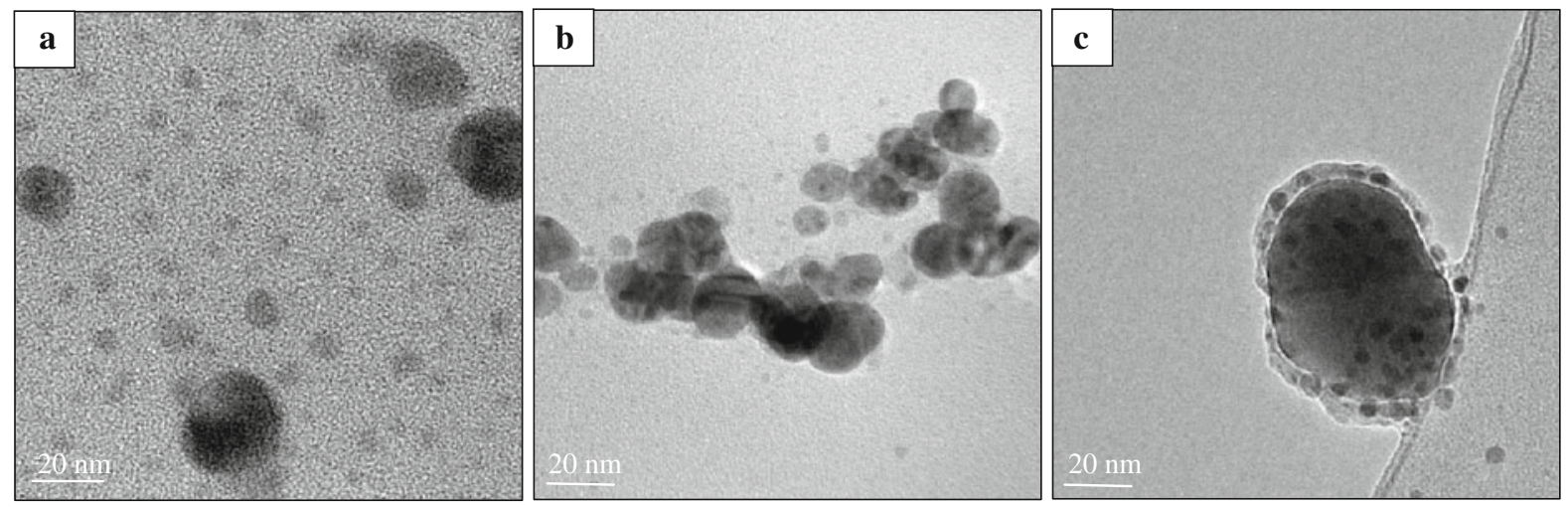

Fig. 3 Examples of TEM images of the silver particles used in the filtration tests: a primary silver particles; b silver agglomerates produced by the coagulation process; $\mathbf{c}$ silver-sintered spheres produced by the sintering process 
Filtration tests of silver agglomerates and sintered spheres

Tests were performed on nanofiber filters composed of a layer of nanofibers on a substrate made of micrometer fibers. Nanofibers can improve the filtration performance compared to conventional filters in certain applications (Wang et al. 2008a, b). The substrate provides necessary support for the fragile nanofiber layer. Effective nanofiber solidity $(\alpha)$, which represents the solid fraction in the nanofiber layer, as well as the nanofiber efficiency $(\%)$ and pressure drop $(\mathrm{Pa})$ were measured and calculated elsewhere (Wang et al. 2008b). We used three layers of the fibrous filter and the face velocity of $5 \mathrm{~cm} / \mathrm{s}$ for all of the experiments presented.

Size distributions as function of mobility diameter of the silver agglomerates upstream (solid line) and downstream (dashed line) of the test filter were measured by SMPS (Fig. 4). As it can be seen from the diagram, the peak is shifted toward bigger particles in the downstream measurements, which is an indication that the chosen test filter is more effective for the smaller particles sizes at the selected face velocity.

Filtration efficiencies of silver-sintered spheres and agglomerates with the same mobility diameters are further on compared in Fig. 5. The slope and position of the efficiency curve for sintered spheres (Fig. 5) is between the curves for $\mathrm{NaCl}$ and agglomerates. The silver spheres have much higher density than $\mathrm{NaCl}$, so the inertial impaction is stronger. The importance of inertial impaction is negligible for small sizes but increases with the size, which qualitatively agrees with the results. It is noticeable that for smaller sizes, up to

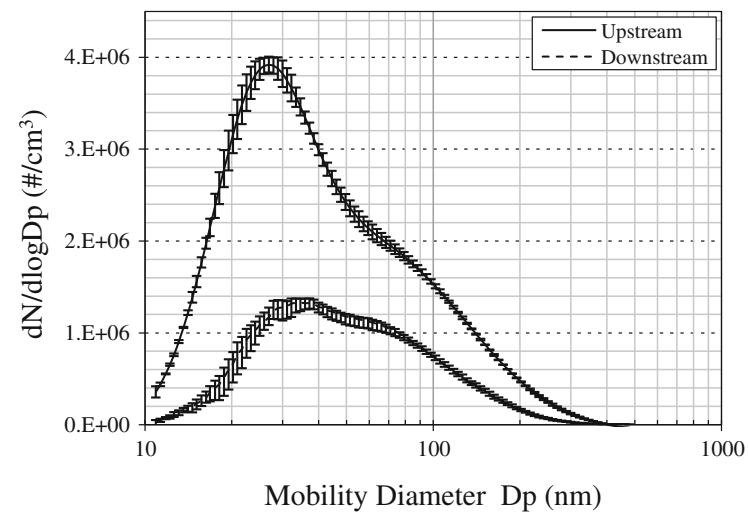

Fig. 4 The size distributions of the silver agglomerates upstream and downstream of the filter
$70 \mathrm{~nm}$, the morphology of the particles does not play a significant role, whereas for bigger sizes the difference becomes more obvious. Diffusion is dominant and interception and inertial impaction have little effects for small particles, thus the particle density and morphology barely influence the filtration efficiency. As the particle size increases, both interception and inertial impaction come back to the play and the difference in the behavior of spherical particles and agglomerates (Fig. 5) becomes more obvious. This is most likely due to the larger interception length in comparison to spherical particles with the same mobility diameter.

\section{Modeling of filtration efficiency}

From the penetration curves presented in Fig. 5, it is obvious that agglomerate particles have lower penetration through filter when compared to spherical particles with the same mobility diameter. The difference in the deposition behavior of agglomerates and sintered spheres is governed by the relationship between the diffusion and interception equivalent diameters for particles with the same density.

Besides the sphere assumption (Fig. 6, dotted line), agglomerate model was used as well (Fig. 6, solid line). We considered diffusion, interception, and inertial impaction to calculate penetration of $\mathrm{Ag}$ agglomerates through the nanofiber filter (Wang et al. 2008b, 2011). Since the penetration $P$ is related to the single fiber efficiency $E$, we chose the following expression for the single fiber efficiency due to the diffusion (Eq. 1),

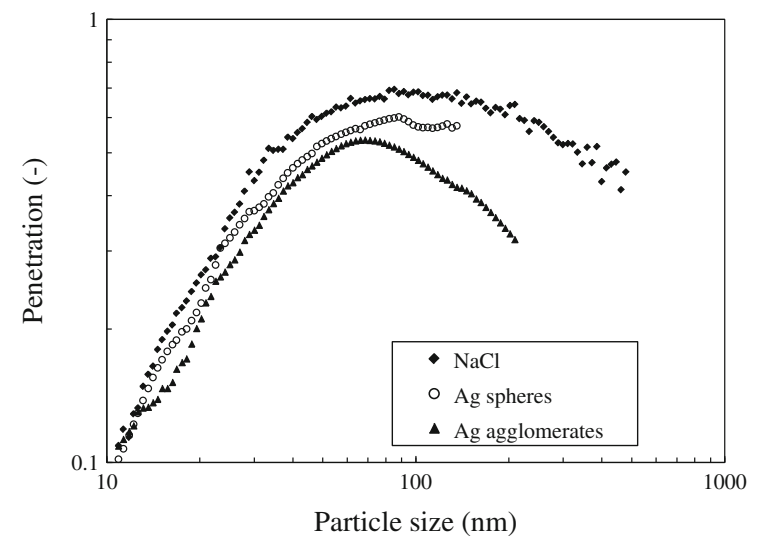

Fig. 5 Penetration curves of polydisperse $\mathrm{NaCl}, \mathrm{Ag}$ sintered spheres and Ag agglomerates 


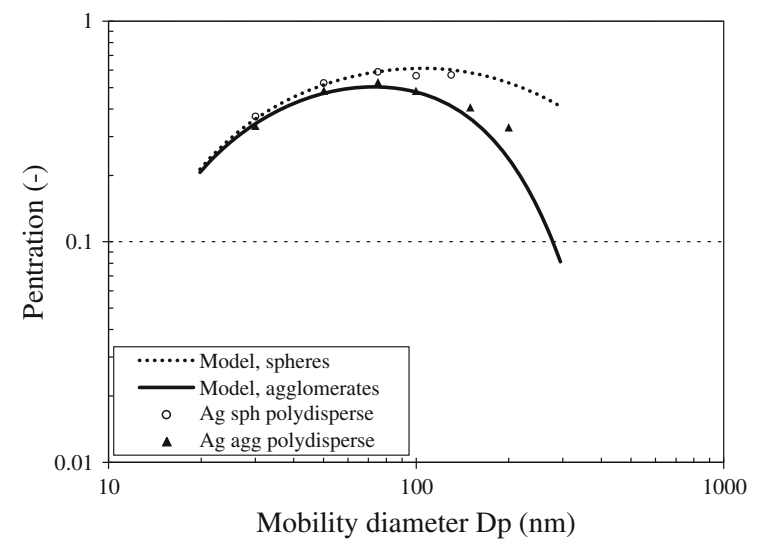

Fig. 6 Penetration curves of polydisperse silver sintered spheres and agglomerates compared with the spheres and agglomerates models, respectively

$E_{\mathrm{D}}=2.27 K_{\mathrm{u}}^{-\frac{1}{3}} P_{\mathrm{e}}^{-\frac{2}{3}}\left(1+0.62 K_{\mathrm{n}} P_{\mathrm{e}}^{\frac{1}{3}} K_{\mathrm{u}}^{-\frac{1}{3}}\right)$,

where $K_{\mathrm{u}}=-\ln \alpha / 2-3 / 4+\alpha-\alpha^{2} / 4$ is the Kuwabara hydrodynamic factor; $P_{\mathrm{e}}=U_{\mathrm{f}} d_{\mathrm{f}} / D$ is the Peclet number with the diffusion coefficient $(D)$, the face velocity of the air incident on the filter $\left(U_{\mathrm{f}}\right)$ and the fiber diameter $\left(d_{\mathrm{f}}\right) ; K_{\mathrm{n}}=2 \lambda / d_{\mathrm{f}}$ is the Knudsen number, $\lambda$ is the mean free path and $\alpha$ is the nanofiber solidity.

The efficiency due to the interception is as follows

$E_{\mathrm{R}}=\frac{(1+R)^{-1}-(1+R)+2\left(1+1.996 K_{\mathrm{n}}\right)(1+R) \ln (1+R)}{2(-0.75-0.5 \ln \alpha)+1.996 K_{\mathrm{n}}(-0.5-\ln \alpha)}$,

For parameter $R$, the equation shown below was used for agglomerates,

$R=B d_{\mathrm{m}}^{\gamma} / d_{\mathrm{f}}$.

where $B$ and $\gamma$ are the factors for the maximum length calculation as reported by Kim et al. (2009a), ( $\gamma$ is found to be 1.268 and $B 0.603$ at room temperature), $d_{m}$ is the mobility diameter.

The efficiencies due to the inertial impaction and interception of particles undergoing diffusion are presented, respectively:

$$
\begin{aligned}
& E_{\mathrm{I}}=\frac{\mathrm{Stk}^{3}}{\mathrm{Stk}^{3}+0.77 \mathrm{Stk}^{2}+0.22}, \\
& E_{\mathrm{DR}}=\frac{1.24 R^{2 / 3}}{\left(K_{\mathrm{u}} P_{\mathrm{e}}\right)^{1 / 2}} .
\end{aligned}
$$

The Stokes number $\left(\mathrm{Stk}=\tau U_{\mathrm{f}} / d_{\mathrm{f}}\right)$ is the measure of inertia. Stk $=\rho d^{2} C_{\mathrm{c}} U_{\mathrm{f}} / 18 \eta d_{\mathrm{f}}$ is the Stokes number for the spherical particles. The relaxation time of the agglomerates is computed based on the mass $(m)$ of the agglomerate reported elsewhere (Kim et al. 2009a). The resulting expression for the Stokes number is given as $\mathrm{Stk}=m C_{\mathrm{c}} U_{\mathrm{f}} /\left(3 \pi \eta d_{\mathrm{m}} d_{\mathrm{f}}\right)$, where $\eta$ is the viscosity and $C_{\mathrm{c}}$ Cunningham slip correction factor.

The total efficiency can further on be computed as

$E=E_{\mathrm{D}}+E_{\mathrm{R}}+E_{\mathrm{I}}+E_{\mathrm{DR}}$,

The penetration of the nanofiber layer is presented as follows,

$P^{\mathrm{N}}=\exp \left(-\frac{4 \alpha E t}{\pi d_{\mathrm{f}}(1-\alpha)}\right)$

where $t$ is the thickness of the nanofiber layer.

To obtain the total penetration for the composite filter, we assume the nanofiber layer and the substrate act independently and in series to capture particles; the penetration through the substrate in the composite filter is the same as that through the bare substrate. Based on these assumptions, we can compute the total penetration as $P=P^{\mathrm{N}} \times P^{\mathrm{S}}$, where $P^{\mathrm{N}}$ and $P^{\mathrm{S}}$ are the penetrations through the nanofiber layer and the substrate, respectively. We compute the $P^{\mathrm{N}}$ from the modeling (Eq. 7) and $P^{\mathrm{S}}$ is assumed to give $94 \%$ penetration for $30 \mathrm{~nm}$ particles and $99 \%$ for all particles that are bigger than $30 \mathrm{~nm}$ (based on the data of Wang et al. 2008a). Out of these results, we can further on obtain $P$, presented in Fig. 6. For the comparison purpose, only selected sizes of polydisperse Ag sintered spheres and Ag agglomerates were chosen out of Fig. 5.

Analyses of the agglomerate filtration data

The SMPS scan results depend on the assumption of the particle morphology. In order to better understand the influence on the filtration data, we have performed the calculations for the number, surface area, and volume of agglomerates based on sphere assumption and open-structured agglomerate assumption.

The number concentration was corrected due to the different charging efficiencies for spheres and agglomerates. The charging efficiency for agglomerates $\left(\eta_{\mathrm{agg}}\right)$ is calculated from Eq. 8 (Wen et al. 1984), where $d_{\mathrm{qe}}$ is 
the charging equivalent diameter (Eq. 9), $e$ is the elementary charge, $T$ is the temperature, $k$ is the Boltzmann constant, $N$ is the primary particle number, and $q$ is the charge on the particle,

$\eta_{\mathrm{agg}}=\frac{e}{\left(\pi d_{\mathrm{qe}} k T\right)^{1 / 2}} \exp \left(\frac{-q^{2} e^{2}}{d_{\mathrm{qe}} k T}\right)$,

$d_{\mathrm{qe}}=\frac{N d}{\ln (2 N)}$.

The number concentration from SMPS, based on sphere, can further on be corrected taking in consideration respective charging efficiencies, as presented in Eq. 10,

$n_{\mathrm{agg}}=n_{\mathrm{sph}} \frac{\eta_{\mathrm{sph}}}{\eta_{\mathrm{agg}}}$.

In addition, for open-structured agglomerates, the surface area may be computed as the sum of surface area of primary particles,

$A_{\text {agg }}=N\left(d_{\mathrm{m}}\right) 4 \pi d^{2}$.

The number of primary particles according to Lall and Friedlander model is shown in Eq. 12, where $c *$ is the dimensionless drag force. Thus, the surface area is independent of the primary particle size (Eq. 13),

$N\left(d_{\mathrm{m}}\right)=\frac{12 \pi \lambda}{c^{*} d^{2}} \frac{d_{\mathrm{m}}}{C_{\mathrm{c}}\left(d_{\mathrm{m}}\right)}$,

$A_{\text {agg }}=N\left(d_{\mathrm{m}}\right)\left(\pi d^{2}\right)=\frac{12 \pi^{2} \lambda}{c *} \frac{d_{\mathrm{m}}}{C_{\mathrm{c}}\left(d_{\mathrm{m}}\right)}$.

The volume of agglomerates may be computed as the sum of volume of primary particles and if the number of primary particles formula is introduced, it shows that it is dependent on the size of the primary particles,

$V_{\text {agg }}=N(d)\left(\frac{1}{6} \pi d^{3}\right)=\frac{2 \pi^{2} \lambda}{c *} \frac{d d_{\mathrm{m}}}{C_{\mathrm{c}}\left(d_{\mathrm{m}}\right)}$.

The same SMPS scan can lead to significantly different volume distributions depending on the assumption of spheres or agglomerates with certain primary size. If the primary particle size increases, the volume increases. Spheres have significantly larger volumes than the agglomerates with the same mobility size (Fig. 7).

In the calculation for filtration efficiency, the upstream and downstream concentrations can be corrected assuming open-structured agglomerates (Eq. 15). The correction factor cancels out and the

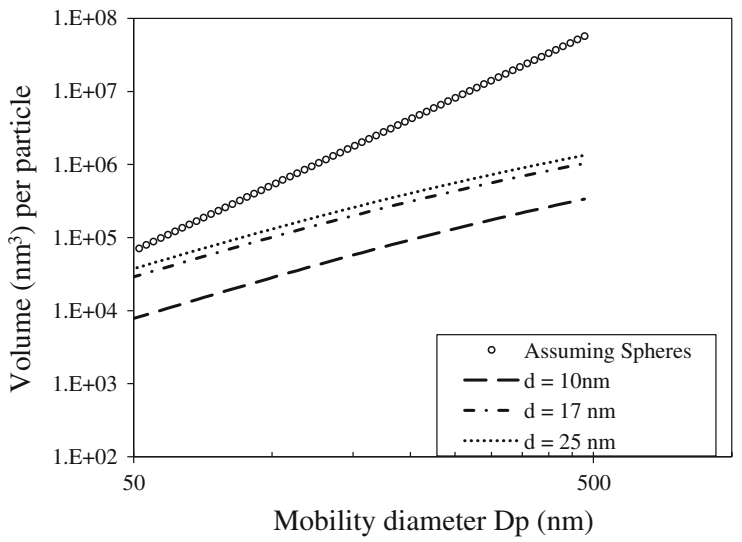

Fig. 7 Volume per particle computed for spheres and for agglomerates for different primary particle size assumption $(10,17$, and $25 \mathrm{~nm})$

filtration efficiency $E_{\text {agg }}$ is the same as computed assuming spheres $E_{\mathrm{sph}}$. The filtration efficiency for surface area or volume is the same as for number. At each mobility diameter, the surface area or volume calculation is the same up- and down-stream and cancels out.

$$
\begin{aligned}
E_{\mathrm{agg}} & =1-\frac{\text { downstream } n_{\mathrm{agg}}\left(d_{\mathrm{m}}\right)}{\operatorname{upstream} n_{\mathrm{agg}}\left(d_{\mathrm{m}}\right)} \\
& =1-\frac{\text { downstream } n_{\mathrm{sph}}\left(d_{\mathrm{m}}\right) \frac{\eta_{\mathrm{sph}}}{\eta_{\mathrm{agg}}}}{\text { upstream } n_{\mathrm{sph}}\left(d_{\mathrm{m}}\right) \frac{\eta_{\mathrm{sph}}}{\eta_{\mathrm{agg}}}}=E_{\mathrm{sph}} .
\end{aligned}
$$

However, the agglomerate correction does affect the filtration efficiency based on total concentrations because the correction is size dependent and its contribution to the total concentration cannot be canceled out. The filtration efficiencies based on total concentrations have wide applications, for example, the NIOSH-standard-compliant filter tester (TSI 8130), uses polydisperse $\mathrm{NaCl}$ particles and a photometer to measure total up- and down-stream concentrations and to evaluate respirator efficiency; the NP surface area monitor (NSAM) has been used to measure up- and down-stream surface area concentrations to evaluate filters (Stanley et al. 2010).

The results regarding the efficiencies based on total concentrations of number, surface area and volume, and the effects by the agglomerate correction are presented in Table 1. If agglomerates with $d=17 \mathrm{~nm}$ give the right filtration efficiency (microscopic study gave the average primary particle size of $16.8 \mathrm{~nm}$ ), the error using the raw SMPS results (spheres) is $\sim 2 \%$ for the number, $3 \%$ for surface area, and $7 \%$ for the volume 
Table 1 Filtration efficiencies computed based on total concentrations of number, surface area and volume

\begin{tabular}{lllll}
\hline Filtration efficiency & Spheres & $\begin{array}{l}\text { Agg with } \\
d=10(\mathrm{~nm})\end{array}$ & $\begin{array}{l}\text { Agg with } \\
d=17(\mathrm{~nm})\end{array}$ & $\begin{array}{l}\text { Agg with } \\
d=25(\mathrm{~nm})\end{array}$ \\
\hline Total number concentration & 0.6373 & 0.6318 & 0.6143 & 0.5905 \\
Total surface area concentration & 0.6118 & 0.5924 & 0.5879 & 0.5831 \\
Total volume concentration & 0.5485 & 0.5924 & 0.5879 & 0.5831 \\
\hline
\end{tabular}

Spheres and open-structured agglomerate assumption with different primary particle sizes used

(graphs not shown here). The results using $d=10,17$, and $25 \mathrm{~nm}$ are very close, which indicates that the value of the primary particle size is not critical for openstructured agglomerates in the calculation of total efficiency. The total efficiency is affected by the agglomerate correction. The effect is weakest for the total number efficiency, and strongest for the total volume efficiency.

We applied multiple charge correction based on sphere assumption to the size distributions of sintered spheres before and after the filter, then the penetrations with and without the multiple charge correction were compared. The maximum difference between the penetrations at each mid-point of the size channel was less than $1.8 \%$; the difference between the total number penetrations was $0.26 \%$. Thus, the effect of multiple charged fractions is negligible for silversintered spheres. The multiple charge fractions for agglomerates are different than spheres, however, they are in the same range (Wen et al. 1984). Thus, we expect the multiple charge fractions have insignificant effect on the penetration calculation.

Filtration efficiency of agglomerates as functions of structure-related parameters

Our results indicate that the agglomerate structure can affect calculation of the filtration efficiency. The primary particle size is an important structural parameter for open-structured agglomerates. The numbersize distribution curve changes with the primary particle size. Since the electrical mobility diameter, as an equivalent diameter, does not describe the morphology of the agglomerate, filtration efficiency for NP agglomerates was additionally plotted as a function of volume equivalent diameter. The penetration results for both silver-sintered spheres and agglomerates are plotted in Fig. 8a. Three curves are presented; the first one using the volume equivalent diameter computed from the open-structured agglomerate model (dashed line), where the primary particle size is assumed to be $17 \mathrm{~nm}$; the second one using the volume equivalent diameter from the sphere assumption (solid line); the third one showing the experimental results for the sintered spheres (dotted line). The electrical mobility diameter and the volume equivalent diameter are the same for spheres. Thus, the curve for sintered spheres in Fig. 8a, is the same as that in Fig. 5. This approach is to illustrate how much the curve is shifted depending on the assumption of the particle morphology. Since the volume equivalent diameter is smaller than the electrical mobility diameter for agglomerates, the curve is shifted to the left. Comparison between the curves for the sintered spheres and agglomerates reveals that for a fixed volume equivalent diameter less than about $40 \mathrm{~nm}$, spheres have smaller mobility diameter, higher diffusivity, therefore lower penetration; for a fixed volume equivalent diameter larger than about $75 \mathrm{~nm}$, agglomerates have larger interception length, therefore lower penetration.

The filtration efficiency is additionally plotted vs. the number of primary particles per agglomerate (Fig. 8b). Since the primary particle size may not be known, three different sizes for primary particle diameter $d(10,17$ and $25 \mathrm{~nm})$ are used. The curve corresponding to $17 \mathrm{~nm}$ represents the correct one. If the primary particle size increases, the number per agglomerate decreases, causing the curve to shift to the left. The choice of the primary particle size has significant effect on the curve.

Primary particle size is essential in data analysis and this information can be provided in different ways. We have chosen to analyze the TEM images and compare it to the SMPS results. Finally ascertained value is further on implemented in the models for the sphere and open-structured agglomerate assumption. 

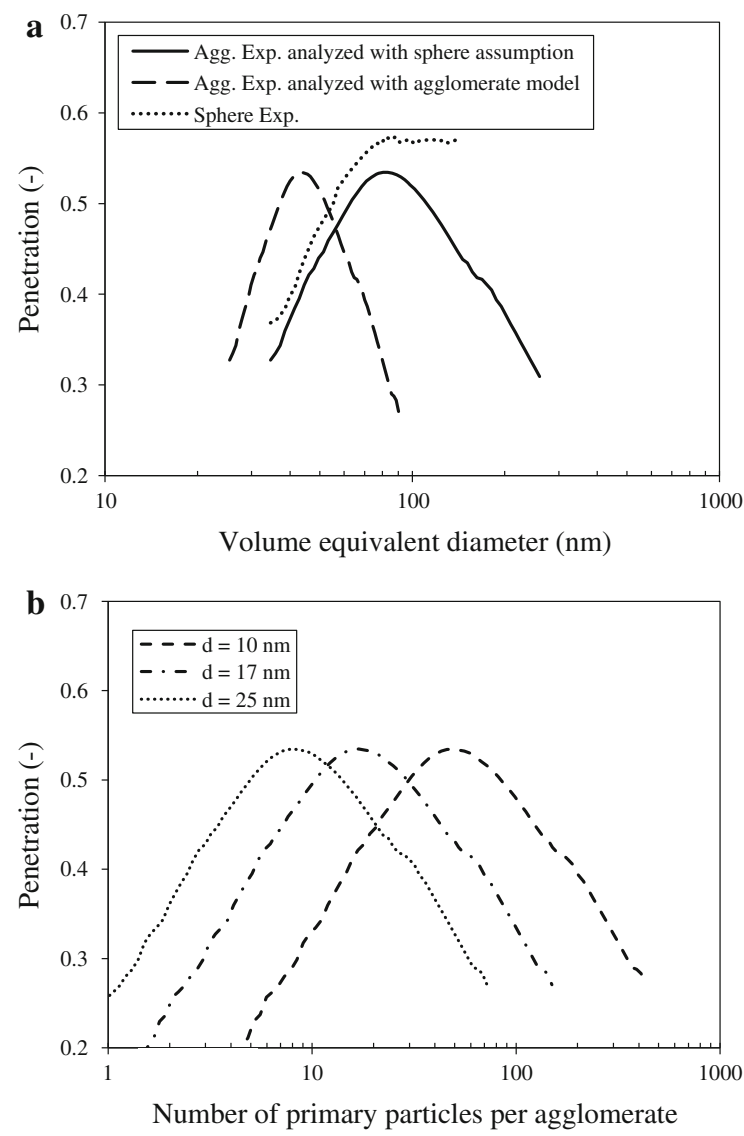

Fig. 8 a Computed filtration penetration curves based on sphere assumption or agglomerate model with $17 \mathrm{~nm}$ particle size and the sphere experiment curve when compared to the volume equivalent diameter; b filtration penetration curves for different primary size particles assumption when compared to the number of primary particles per agglomerate

\section{Conclusions}

Our study shows that agglomerate particles have lower penetration through filter when compared to spherical particles with the same mobility diameter. For smaller particles, diffusion dominates, sintered spheres, and agglomerates have similar filtration. For bigger sizes, interception becomes important; agglomerates have extended branches, therefore lower penetration. By sintering the agglomerates to a spherical shape, we were able to obtain a direct comparison of nanoparticle agglomerate filtration to compact structure nanoparticles of the same material. When the particle morphology is not known a priori, the filtration data may be processed with different assumptions. The curve of filtration efficiency versus mobility size is not affected by the assumption on particle morphology. However, the efficiency based on total concentrations is affected by the agglomerate correction. The effect is the weakest for the total number efficiency, and the strongest for the total volume efficiency. The filtration efficiency of open-structured agglomerates as a function of geometric parameters, such as volume per agglomerate, can only be characterized with information of the primary particle size. Primary particle size was ascertained with both SMPS and TEM. Such filtration efficiency data provide insight for the effect of particle structure on filtration.

Acknowledgments Financial support by the European Committee for Standardization in the frame of mandate M/461 "Standardization activities regarding nanotechnologies and nanomaterials" is acknowledged. The authors also thank the support of members of the Center for Filtration Research: 3M, Boeing Commercial Airplanes, Cummins Filtration, Donaldson Co., Inc., Entegris, Inc., Hollingsworth \& Vose Co., MANN + HUMMEL, GMBH, MSP Corp., Samsung Electronics Co., Ltd, Shigematsu Works Co., Ltd, TSI Inc., and W. L. Gore \& Associates, Inc. and affiliate member NIOSH.

\section{References}

Cai J, Sorensen CM (1994) Diffusion of fractal aggregates in the free molecular regime. Phys Rev E 50(5):3397-3400

Cena LG, Ku BK, Peters TM (2012) Particle collection efficiency for nylon mesh screens. Aerosol Sci Technol 46(2):214-221. doi:10.1080/02786826.2011.617401

Fu T-H, Cheng M-T, Shaw DT (1990) Filtration of chain aggregate aerosols by model screen filter. Aerosol Sci Technol 13(2):151-161. doi:10.1080/02786829008959433

Japuntich DA, Franklin LM, Pui DY, Kuehn TH, Kim S, Viner AS (2007) A comparison of two nano-sized particle air filtration tests in the diameter range of 10 to 400 nanometers. J Nanopart Res 9:93-107. doi:10.1007/978-1-40205859-2_10

Kim SC, Wang J, Emery MS, Shin WG, Mulholland GW, Pui DYH (2009a) Structural property effect of nanoparticle agglomerates on particle penetration through fibrous filter. Aerosol Sci Technol 43(4):344-355. doi:10.1080/ 02786820802653763

Kim SC, Wang J, Shin WG, Scheckman JH, Pui DYH (2009b) Structural properties and filter loading characteristics of soot agglomerates. Aerosol Sci Technol 43(10):1033-1041. doi:10.1080/02786820903131081

Kousaka Y, Endo Y, Ichitsubo H, Alonso M (1996) Orientationspecific dynamic shape factors for doublets and triplets of spheres in the transition regime. Aerosol Sci Technol 24(1):36-44

$\mathrm{Ku}$ BK, Maynard AD (2006) Generation and investigation of airborne silver nanoparticles with specific size and morphology by homogeneous nucleation, coagulation and sintering. J Aerosol Sci 37(4):452-470. doi:10.1016/j.jaerosci.2005.05.003 
Lall AA, Friedlander SK (2006) On-line measurement of ultrafine aggregate surface area and volume distributions by electrical mobility analysis: I. Theoretical analysis. J Aerosol Sci 37(3):260-271. doi:10.1016/j.jaerosci.2005.05.021

Lall AA, Seipenbusch M, Rong W, Friedlander SK (2006) Online measurement of ultrafine aggregate surface area and volume distributions by electrical mobility analysis: II. comparison of measurements and theory. J Aerosol Sci 37(3):272-282. doi:10.1016/j.jaerosci.2006.01.006

Lange R, Fissan H, Schmidt-Ott A (1999) Predicting the collection efficiency of agglomerates in fibrous filters. Part Part Syst Charact 16(2):60-65. doi:10.1002/(sici)15214117(199906)16:2<60:aid-ppsc60>3.0.co;2-a

Lange R, Fissan H, Schmidt-Ott A (2000) A new method for the determination of the interception equivalent diameter. J Aerosol Sci 31(2):221-231. doi:10.1016/s0021-8502(99)00050-6

Oberdörster G, Oberdörster E, Oberdörster J (2005) Nanotoxicology: an emerging discipline evolving from studies of ultrafine particles. Environ Health Perspect 113(7):823839. doi:10.1289/ehp.7339

Park K, Kittelson DB, McMurry PH (2004) Structural properties of diesel exhaust particles measured by transmission electron microscopy (TEM): relationships to particle mass and mobility. Aerosol Sci Technol 38(9):881-889. doi: $10.1080 / 027868290505189$

Schmidt-Ott A (1988) New approaches to in situ characterization of ultrafine agglomerates. J Aerosol Sci 19(5):553563. doi:10.1016/0021-8502(88)90207-8

Shin W, Wang J, Mertler M, Sachweh B, Fissan H, Pui D (2009a) Structural properties of silver nanoparticle agglomerates based on transmission electron microscopy: relationship to particle mobility analysis. J Nanopart Res 11(1):163-173. doi:10.1007/s11051-008-9468-y

Shin WG, Mulholland GW, Kim SC, Wang J, Emery MS, Pui DYH (2009b) Friction coefficient and mass of silver agglomerates in the transition regime. J Aerosol Sci 40(7):573-587. doi:10.1016/j.jaerosci.2009.02.006

Shin WG, Wang J, Mertler M, Sachweh B, Fissan H, Pui DYH (2010) The effect of particle morphology on unipolar diffusion charging of nanoparticle agglomerates in the transition regime. J Aerosol Sci 41(11):975-986. doi:10.1016/ j.jaerosci.2010.07.004
Song CB, Park HS (2006) Analytic solutions for filtration of polydisperse aerosols in fibrous filter. Powder Technol 170(2):64-70. doi:10.1016/j.powtec.2006.08.011

Song DK, Lenggoro IW, Hayashi Y, Okuyama K, Kim SS (2005) Changes in the shape and mobility of colloidal gold nanorods with electrospray and differential mobility analyzer methods. Langmuir 21(23):10375-10382. doi:10.1021/ la0513196

Sorensen CM (2011) The mobility of fractal aggregates: a review. Aerosol Sci Technol 45(7):765-779. doi:10.1080/ 02786826.2011.560909

Stanley N, Qi C, Pui DYH (2010) a new method of filter efficiency evaluation using the nanoparticle surface area monitor (NSAM) for a nanoparticle health relevant filter efficiency measure. Filtration 10(1):40-46

Wang GM, Sorensen CM (1999) Diffusive mobility of fractal aggregates over the entire knudsen number range. Phys Rev E 60(3):3036-3044

Wang J, Kim SC, Pui DYH (2008a) Figure of merit of composite filters with micrometer and nanometer fibers. Aerosol Sci Technol 42(9):722-728. doi:10.1080/02786820802 249133

Wang J, Kim SC, Pui DYH (2008b) Investigation of the figure of merit for filters with a single nanofiber layer on a substrate. J Aerosol Sci 39(4):323-334. doi:10.1016/j.jaerosci.2007. 12.003

Wang J, Shin WG, Mertler M, Sachweh B, Fissan H, Pui DYH (2010) Measurement of nanoparticle agglomerates by combined measurement of electrical mobility and unipolar charging properties. Aerosol Sci Technol 44(2):97-108. doi:10.1080/02786820903401427

Wang J, Kim SC, Pui DYH (2011) Carbon nanotube penetration through a screen filter: numerical modeling and comparison with experiments. Aerosol Sci Technol 45(3):443-452. doi:10.1080/02786826.2010.541531

Wen HY, Reischl GP, Kasper G (1984) Bipolar diffusion charging of fibrous aerosol particles-I. Charging theory. J Aerosol Sci 15(2):89-101. doi:10.1016/0021-8502(84) 90029-6

Zelenyuk A, Imre D (2007) On the effect of particle alignment in the DMA. Aerosol Sci Technol 41(2):112-124. doi:10.1080/ 02786820601118380 